\title{
Concepts for fast acquisition in optical communications systems
}

\author{
Brandon L. Wilkerson ${ }^{\mathrm{a}}$, Dirk Giggenbach*º ${ }^{\mathrm{b}}$, Bernhard Epple ${ }^{\mathrm{b}}$ \\ ${ }^{\mathrm{a} U}$ US. Air Force, German Aerospace Center (DLR), Wessling, Germany; \\ ${ }^{\mathrm{b}}$ German Aerospace Center (DLR), Institute of Communications \& Navigation, Wessling, Germany
}

\begin{abstract}
Abstract-As free-space laser communications systems proliferate due to improved technology and transmission techniques, optical communication networks comprised of ground stations, aircraft, high altitude platforms, and satellites become an attainable goal. An important consideration for optical networks is the ability of optical communication terminals (OCT) to quickly locate one another and align their laser beams to initiate the acquisition sequence. This paper investigates promising low-cost technologies and novel approaches that will facilitate the targeting and acquisition tasks between counter terminals. Specifically, two critical technology areas are investigated: position determination (which includes location and attitude determination) and inter-terminal communications. A feasibility study identified multipleantenna global navigation satellite system (GNSS) systems and GNSS-aided inertial systems as possible position determination solutions. Personal satellite communication systems (e.g. Iridium or Inmarsat), third generation cellular technology (IMT-2000/UMTS), and a relatively new air traffic surveillance technology called Autonomous Dependent Surveillance-Broadcast (ADS-B) were identified as possible inter-terminal communication solutions. A GNSS-aided inertial system and an ADS-B system were integrated into an OCT to demonstrate their utility in a typical optical communication scenario. Testing showed that these technologies have high potential in future OCTs, although improvements can be made to both to increase tracking accuracy.
\end{abstract}

Keywords: Optical communication terminals, Satellite mobile communication, Satellite navigation systems, Position measurement, Attitude determination, Pointing Systems, ADS-B

\section{INTRODUCTION}

The work presented in this paper partially relates to the ATENAA project [30], funded by the European Union, aiming to establish broadband wireless optical communication for commercial aviation.

Optical communication has the potential to increase data rates while using less power and weighing less than traditional RF communication systems. One important development to make this possible is the ability for optical communication terminals (OCT) to autonomously locate and acquire a counter terminal.

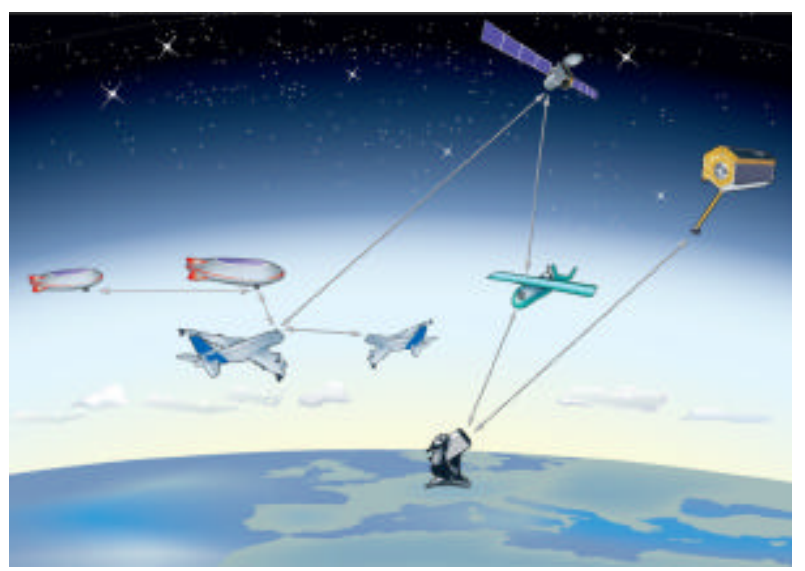

Fig. 1. Example of a future optical communication network made up of optical communication terminals (OCT).

*dirk.giggenbach@dlr.de; phone 498153282821

Copyright 2006 Society of Photo-Optical Instrumentation Engineers. This paper was published in Proceedings of the SPIE Vol. 6304 and is made available as an electronic reprint with permission of SPIE. One print or electronic copy may be made for personal use only. Systematic or multiple reproduction, distribution to multiple locations via electronic or other means, duplication of any material in this paper for a fee or for commercial purposes, or modification of the content of the paper are prohibited. 
This paper investigates potential technologies and techniques that will enable rapid acquisition between two OCTs and that can be integrated into low-cost and lightweight pointing, acquisition, and tracking (PAT) systems of terrestrial and aeronautical OCTs. These OCTs will make up optical communications networks, such as the example pictured in Fig. 1.

Several examples of OCTs from the Capanina project are shown in Fig. 2. These terminals successfully demonstrated a $64 \mathrm{~km}$ aeronautical optical communication link in August $2005[1,2]$
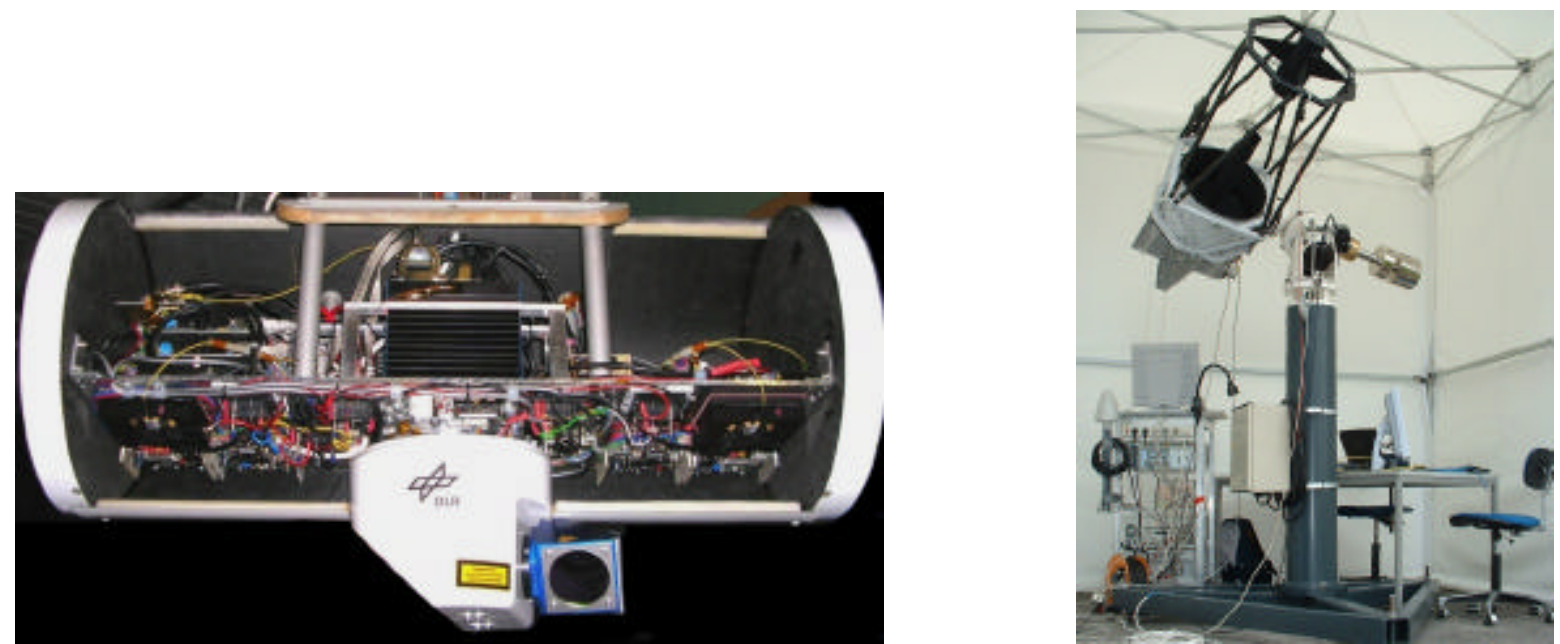

Fig. 2 Left: Optical terminal that flew on a stratospheric balloon in Capanina trial. The coarse pointing assembly is a periscope located on the bottom of the terminal. Right: Optical ground station used for Capanina trial.

To initiate an optical communication link between two OCTs, both terminals must simultaneously accomplish the following steps:

1. Have knowledge that a counter terminal is in communication range.

2. Determine own position (location and attitude) information.

3. Communicate own position to counter terminal and receive counter terminal's position information.

4. Calculate a pointing vector to the counter terminal based on the position information of each terminal.

5. Calculate the required attitude change of the coarse pointing assembly to align it with the pointing vector and then carry out the alignment.

These steps must occur quickly and continuously until both terminals are aligned accurately enough to initiate optical tracking. Optical tracking is necessary to achieve the required pointing accuracy due to the small divergence angles of the communication lasers and is done by means of a beacon laser or the actual communication laser. If the optical tracking link is broken for any reason, it may be necessary to perform the location and acquisition steps again. Although this acquisition process is not unique to optical communications scenarios, the required accuracy is higher than in other scenarios. The challenge addressed in this paper is finding low-cost and lightweight solutions that can be integrated into OCT PAT systems that meet this accuracy requirement.

When considering the pointing, acquisition, and tracking tasks, multiple factors degrade the system accuracy and performance. These factors will be discussed in Section 2, highlighting the two important performance characteristics for OCT PAT systems: position measurement (determination of both location and attitude) and inter-terminal communications. The results of a feasibility study, which focused on available hardware and techniques relating to these two topics, are presented in Section 3 and Section 4. Based on the results of the feasibility study, a position measurement system and a communication system were tested by integrating them into optical terminals and demonstrating their utility during tracking tests. The results from these tests as well as ways to increase performance are presented in Section 5. 


\section{POINTING AND TRACKING ACCURACY DISCUSSION}

When implementing a pointing and tracking (PAT) system in optical communication terminals (OCT), it is impossible to create a perfect system. Several factors degrade the pointing accuracy of the OCT and hinder its ability to precisely track a target. The causes of this accuracy degradation can be broken into two categories: uncertainty errors in the PAT system and time delays in the inter-terminal communication system. Although a complete discussion of these two categories is beyond the scope of this paper, each of these categories will be discussed within the framework of OCT scenarios. Table 1 lists the primary contributing factors that lead to a degradation of pointing and tracking accuracy in OCTs. These factors are discussed in Section 2.1 and Section 2.2.

Table 1. Factors leading to the degradation of pointing and tracking accuracy

\begin{tabular}{|c|c|}
\hline PAT system uncertainty errors & Communication system time delays \\
\hline \hline Coarse pointing attitude knowledge & Transmission rate \\
Coarse pointing resolution & Transmission delay \\
Receiver position measurement & Internal calculation time \\
Transmitter position measurement & \\
\hline
\end{tabular}

\subsection{Uncertainty errors}

The PAT system uncertainty errors listed in Table 1 effectively result in an overall pointing error. This overall error is depicted in Fig. 3, which shows a simple optical communication scenario between a ground station and a mobile terminal. If the errors in the ground station PAT system are large enough, the ground station will not "see" the mobile terminal and will not be able to initiate an optical communication link.

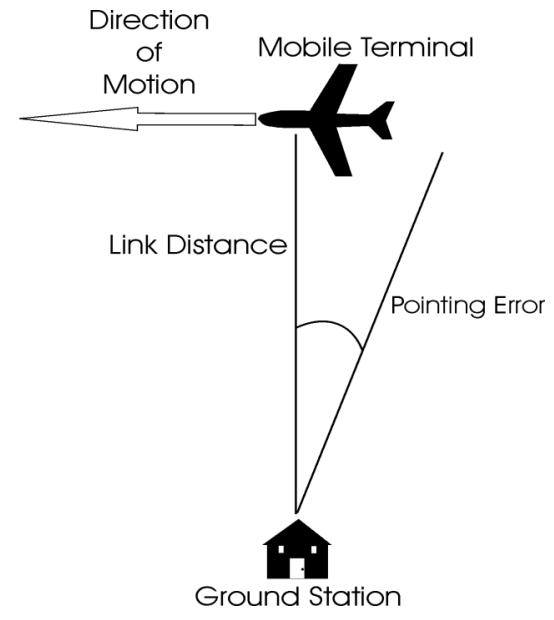

Fig. 3. Representative optical communication scenario between a ground station and mobile airborne terminal

The coarse pointing attitude knowledge is the ability of the pointing assembly to know exactly where it's pointed whereas the coarse pointing resolution is the smallest pointing change that the assembly can make. The coarse pointing assembly uncertainty errors are usually a factor of the selection and mechanics of the motors, gears, encoders, and structure. Normally, as the quality (and weight) of the components increases, the errors decrease and the cost increases. These errors are not discussed in great detail in this paper, however [3] provides a more detailed analysis.

Position measurement is the determination of both the location and attitude of the OCT. It can be accomplished using various sensors and techniques; however, this paper takes a limited approach considering only solutions which are based upon global navigation satellite systems (GNSS) and/or inertial systems. Section 3 discusses position measurement in more depth. 


\subsection{Time delays}

In order to locate a counter terminal and initiate the acquisition sequence, the terminals must be able to communicate with some outside source. In this paper, it is assumed that the terminals will communicate directly with one another. All communication systems have inherent time delays, which in the case of OCTs can reduce the accuracy of the PAT system. In most optical communication scenarios, one or both OCTs are moving which adds complexity to the communication system. One characteristic that differentiates time delays from uncertainty errors is that time delays can be measured and accounted for, which will be further discussed in Section 5.

The transmission rate is how often each successive update of the counter terminal position information. Generally, short link distances and large relative velocities between the terminals require a higher transmission rate. If the rate is not high enough, the PAT system can not react quickly enough to keep up with the moving terminal.

The transmission delay is the time it takes for the position information to get from the transmitter to the receiver. In a line of sight RF communication system, this delay time is extremely short. On the other hand, if a satellite link or a cellular link is used, the transmit delay can be large. Again, larger time delays increase the chance that the PAT system is not fast enough to track the target.

The last time delay source is internal calculation time. This is the time necessary to collect and calculate position data, format the data for transmission, and process the data once it has reached the receiver. This time delay is much smaller than the other two sources of delay and won't be discussed in detail in this paper.

The applicable time delays for various communication systems are discussed in Section 4.

\section{POSITION MEASUREMENT}

\subsection{Background}

As stated earlier, position measurement includes both location measurement and attitude measurement. The use of a GNSS is clearly the best solution for location measurement. Low cost off-the-shelf receivers can be used and their accuracy is ever increasing. Europe is currently developing the Galileo Global Navigation Satellite System which, according to a 2004 agreement between the United States and the European Union, will be fully compatible and interoperable with GPS [1]. This promises increased integrity and accuracy of GNSS solutions. Additionally, the implementation of Satellite Based Augmentation Systems (SBAS) has significantly increased the accuracy, reliability, and integrity of GNSS. Two well known SBAS systems are the Wide Area Augmentation System (WAAS) in the US and the European Geostationary Navigation Overlay System (EGNOS). Is should be noted that EGNOS will be part of the implemented Galileo architecture. Japan and India are also developing satellite based augmentation systems. Typical GNSS (GPS and Galileo) accuracies are summarized in Table 2.

Table 2. GNSS Accuracies [5,6]

\begin{tabular}{|l|l|}
\hline $\begin{array}{l}\text { Original GPS (subject to Selective Availability } \\
\text { accuracy degradation) }\end{array}$ & $100 \mathrm{~m}$ \\
\hline GPS without Selective Availability & $15 \mathrm{~m}$ \\
\hline Differential GPS (DGPS) position accuracy & $3-5 \mathrm{~m}$ \\
\hline WAAS & $<3 \mathrm{~m}$ \\
\hline $\begin{array}{l}\text { Galileo Commercial Service (advertised); global } \\
\text { usage (i.e. without local augmentation) }\end{array}$ & $<1 \mathrm{~m}$ \\
\hline
\end{tabular}

Since a GNSS receiver is a necessary component in an OCT, leveraging this hardware for the attitude determination solution may be an intelligent design decision. For the attitude portion of position measurement, the following three approaches are investigated: 1) Multiple-antenna GNSS attitude determination; 2) Inertial system attitude determination (GNSS-aided, single antenna); and 3) Inertial system attitude determination (GNSS-aided, dual antenna) 


\subsection{Multiple-antenna GNSS Attitude Determination [7]}

During the past 15 years, research has shown that GPS-based attitude determination can offer precision attitude knowledge. This solution is based on measuring the difference in the carrier phase between two antennas. With 3 or more antennas, receivers can estimate the attitude of the vehicle. Off-the-shelf systems usually operate with 4 antennas and 4 separate receivers built into a common housing. The precision of such systems is dependent upon the spacing between the antennas, called the baseline. The following equation gives a rough estimate of the attitude determination angular accuracy for a given baseline length, $\mathrm{L}$.

$$
\sigma_{\theta}(\text { in radians }) \cong \frac{0.5 \mathrm{~cm}}{L(\text { incm })}
$$

With a 1 meter baseline, several surveyed off-the-shelf systems offer better than $1^{\circ}$ accuracy in the roll, pitch, and yaw (heading) axes. The characteristics of two candidate systems from Thales Navigation and Septentrio Satellite Navigation are listed in Table 3.

\subsection{GNSS-aided Inertial System Solution (Single Antenna)}

Another option for the attitude solution is a GPS aided inertial system. A standalone inertial measurement unit (IMU) can be used to provide the attitude information, but there are advantages of using a GPS-aided system. Purely inertial systems can drift or diverge over time and the problem is worse with low-cost systems. Using GPS to update the inertial system maintains an accurate solution. During periods of GPS outage (due to antenna blanking or system outages), the inertial system can continue providing accurate information [8]. The two systems, in effect, complement one another. Since a GNSS position solution is needed in any case, it is logical to consider a GPS-aided inertial system. Affordable off-the-shelf strap-down inertial system models are available today. These systems use MEMS-based inertial sensors to provide attitude and heading information. When aided by a GPS solution, these systems can give accurate position, velocity and attitude information. The Crossbow NAV420 GPS-aided inertial system is a candidate system and is listed in Table 3.

\subsection{GNSS-aided Inertial System Solution (Dual Antenna)}

Low-cost inertial systems often lack the ability to provide an accurate heading measurement, even with the system is GPS aided. To overcome this relatively low heading accuracy, it is possible to take advantage of the concept of multiple GNSS antennas along with an inertial system. A two antenna system can increase the heading accuracy when coupled with a strapdown inertial system. This concept was demonstrated by the University of Nottingham in a marine surveying experiment by integrating a two antenna GPS receiver and an inertial measurement system, both of which were off the shelf products. In doing so, the heading error was reduced by about $80 \%$ compared to a single antenna GPS receiver [9]. Whereas market research turned up several stand-alone dual-GPS antenna heading systems, only a few inertial systems aided by dual-antenna GPS were found. Enpoint, a small company in the US, is developing a system that could offer $0.4^{\circ}$ (7 mrad) accuracy for about $\$ 6000$ [10]. A standalone dual-antenna system from Javad Navigation Systems is listed in Table 3

Table 3. Specification overview of various GNSS position determination systems $[11,12,13,14]$

\begin{tabular}{|c|c|c|c|c|}
\hline & $\begin{array}{l}\text { Thales ADU-5 } \\
\text { (GNSS only) }\end{array}$ & $\begin{array}{l}\text { Septentrio PolaRx2 } \\
\text { (GNSS only) }\end{array}$ & $\begin{array}{l}\text { Crossbow NAV420 } \\
\text { (GPS aided inertial) }\end{array}$ & $\begin{array}{c}\text { JNS Gyro2 } \\
\text { (Dual Antenna GPS) }\end{array}$ \\
\hline Cost (receivers \& antennas) & $\$ 20,000$ & $\$ 18,000$ & $\$ 15,000$ & $\$ 25,000$ \\
\hline $\begin{array}{l}\text { Position Accuracy (horizontal/vertical) } \\
\text { Standalone } \\
\text { SBAS } \\
\text { DGPS }\end{array}$ & $\begin{array}{c}\text { (CEP values) } \\
3 \mathrm{~m} \\
1.8 \mathrm{~m} \\
0.9 \mathrm{~m}\end{array}$ & $\begin{array}{l}\text { (1s values) } \\
1.1 \mathrm{~m} / 1.9 \mathrm{~m} \\
0.7 \mathrm{~m} / 1.2 \mathrm{~m} \\
0.6 \mathrm{~m} / 1.1 \mathrm{~m}\end{array}$ & $\begin{array}{l}\text { (CEP values) } \\
3 \mathrm{~m}\end{array}$ & $\begin{array}{c}\text { (RMS Values) } \\
3 \mathrm{~m}\end{array}$ \\
\hline $\begin{array}{l}\text { Attitude Accuracy (heading / pitch,roll) } \\
1 \mathrm{~m} \text { baseline }\end{array}$ & $\begin{array}{l}0.4^{\circ} / 0.8^{\circ} \\
7 / 14 \mathrm{mrad}\end{array}$ & $\begin{array}{l}0.3^{\circ} / 0.6^{\circ} \\
5 / 10 \mathrm{mrad}\end{array}$ & $\begin{array}{c}3.0^{\circ} / 0.75^{\circ} \\
52 / 13 \mathrm{mrad}\end{array}$ & $\begin{array}{l}0.3^{\circ} /-- \\
5 \mathrm{mrad}\end{array}$ \\
\hline Output rate & $1-5 \mathrm{~Hz}$ & $1-10 \mathrm{~Hz}$ & $2-100 \mathrm{~Hz}$ & $20 \mathrm{~Hz}$ \\
\hline GNSS Channels & 56 & 48 & 16 & 20 \\
\hline SBAS Compatible & Yes & Yes & Yes (WAAS) & Yes (WAAS) \\
\hline Number of antennas & 4 & 3 & 1 & 2 \\
\hline Mass (receiver / antennas) & $1.93 / 0.46 \mathrm{~kg}$ & $0.75 / ? ? \mathrm{~kg}$ & $0.58 \mathrm{~kg}$ (total) & $0.795 \mathrm{~kg}$ \\
\hline Power & $<6$ watt & 5-7 watt & $<5$ watt & 4.2 watt \\
\hline
\end{tabular}




\section{INTER-TERMINAL COMMUNICATION TECHNOLOGIES}

\subsection{Background}

Although numerous communication systems exist that could provide a viable inter-terminal communication solution, three techniques in particular will be considered. These include:

- Current Personal Satellite Communication systems

- IMT-2000 Mobile Satellite Service (MSS)

- Automatic Dependent Surveillance-Broadcast

\subsection{Global Mobile Personal Communication by Satellite}

Personal Satellite Communication systems include systems such as Inmarsat, Iridium, Globalstar, and Orbcomm. These systems take different approaches with respect to satellite constellations, data rates, coverage areas, and services. The user segments of these systems operate predominantly in L-band (e.g. Inmarsat: $1525-1529 \mathrm{MHz}$ ).

These systems use higher frequencies than $3 \mathrm{G}$ cell phones and have not offered the same advanced $3 \mathrm{G}$ services since they were developed earlier. However, GMPCS systems are becoming more popular and more widely used. Capabilities are expanding and a wider range of services are being offered, such as packet switched data and "always on" connections.

This paper focuses on the Inmarsat and Iridium systems. Inmarsat is considered due to the services and data rates offered and Iridium due to its near-global coverage. The technical details of the Inmarsat and Iridium systems are discussed further in [15]. Each of these systems offers various terminal options ranging from vehicle-mounted terminals to handsets. Additionally, both systems offer a data service, which is most applicable to inter-terminal communications. In some cases, the data service comes as part of the terminal (e.g. Inmarsat GAN) and in other cases a data kit must be used to feed data from a computer to the terminal (e.g. RS-232 data kit for the Iridium systems). Table 4 gives an overview of several promising terminals that could be considered for inter-terminal communications.

Table 4. Overview of Personal Satellite Communication system options [16]

\begin{tabular}{|l|l|l|l|l|}
\hline & Nera NWC Voyager & Nera Worldphone Voyager & SkyConnect Mobile & 9505A Portable Phone \\
\hline Constellation & Inmarsat & Inmarsat & Iridium & Iridium \\
\hline Service & GAN/M4 & Mini-M & -- & -- \\
\hline Price & $\$ 14,835$ & $\$ 4,552$ & $\$ 2,995$ & $\$ 1,395$ \\
\hline Use & Vehicle Mobile & Vehicle Mobile & Vehicle Mobile & Portable \\
\hline Data Rate & $64 \mathrm{Kbps}$ & $2.4 \mathrm{Kbps}$ & $2.4 \mathrm{Kbps}$ & $2.4 \mathrm{Kbps}$ \\
\hline Weight & $15 \mathrm{~kg}$ & $5.6 \mathrm{~kg}$ & $2 \mathrm{~kg}$ & Handset \\
\hline Antenna Size $(\mathrm{cm})$ & $75 \times 71 \times 38$ & $14 \times 27 \times 27$ & $9 \times 9 \times 2$ & handset \\
\hline Terminal Size $(\mathrm{cm})$ & $31 \times 18 \times 8$ & $80 \times 24 \times 20$ & $27 \times 12 \times 7$ & handset \\
\hline Speed capability & $250 \mathrm{~km} / \mathrm{hr}$ & $250 \mathrm{~km} / \mathrm{hr}$ & Aircraft compatible & -- \\
\hline Turning capability & $40 \% \mathrm{~s}$ & $60 \% \mathrm{~s}$ & Aircraft compatible & -- \\
\hline
\end{tabular}

Iridium satellite modems have already been proven in aeronautical applications [17,18]. In an OCT PAT scenario, communication between two terminals would be achieved using data modems on each terminal communicating over the Personal Satellite Communication system. The advantages of such systems are the near global coverage and the high transmission rate possible using a packed switched system. The disadvantage is the transmission delay which can range from 0.1 second (speed of light transmission time to a LEO satellite) to multiple seconds if the packet is lost or delayed.

\subsection{IMT-2000 Mobile Satellite Service [19]}

The International Telecommunications Union sponsored a project called International Mobile Telecommunications 2000 (IMT-2000) and gave this name to the worldwide 3G cellular standard that was developed by the project. The IMT-2000 
standard in Europe is known as Universal Mobile Telecommunication System (UMTS). Different world markets have taken various implementation approaches (e.g. UMTS in Europe and CDMA2000 in the US), often resulting in incompatible frequencies and systems. However, in addition to the $3 \mathrm{G}$ cellular system, there is a $3 \mathrm{G}$ satellite component called Mobile Satellite Service (MSS), which is envisioned to be an extension of the 3G cellular systems. And although different frequencies exist for $3 \mathrm{G}$ cellular, most countries have allocated the same frequencies for MSS (2000 MHz range). The concept is that a user could use a $3 \mathrm{G}$ handset outside of the cellular coverage by use of the MSS. This segment is sometimes referred to as Space UMTS (S-UMTS).

There are currently no satellite systems on orbit to support MSS, but [20] and [21] provide promising details that MSS systems that are compatible with $3 \mathrm{G}$ cell phones may be realized in the near future. Assuming that MSS systems will operate like GMPCS systems, there will be similar hardware solutions (i.e. data modems) in the future. Land-based 3G systems are already available offering $384 \mathrm{Kbps}$ with standard air time or data volume tariffs [22]. If MSS systems become reality, the data rates will be approximately $100 \mathrm{Kbps}$ [19]. This system could again provide worldwide coverage at potentially lower costs than the Personal Satellite Communication systems, but would have the same time delay disadvantages.

\subsection{Automatic Dependent Surveillance-Broadcast}

Another concept that could be used for inter-terminal communications is based on a relatively recent air traffic control (ATC) surveillance concept called Automatic Dependent Surveillance - Broadcast (ADS-B), which is an airborne data link based on GNSS technology. An ADS-B capable aircraft uses a GNSS receiver to determine its precise position and then broadcasts its position along with other important information (e.g. identification, speed, ac) [23]. Any other vehicle or ground station within range that has an ADS-B receiver can receive the real-time ADS-B data.

Currently, there are three different types of ADS-B links: 1) $1090 \mathrm{MHz}$ Mode S Extended Squitter (ES); 2) universal access transceiver (UAT); and 3) VHF data link (VDL) Mode 4 [24]. Of the 3, only $1090 \mathrm{MHz}$ Mode S ES has spectrum approval for global operation [25]. ADS-B enabled hardware is starting to show up on the market. Several systems are summarized in Table 5.

Table 5. Overview of ADS-B System Options [26,27,28]

\begin{tabular}{|l|l|l|l|}
\hline & Garmin GDL 90 & Filser TRG80 & C.N.S Systems VDL 4000/GA \\
\hline Type & Universal Access Transceiver & Mode S Extended Squitter & VDL Mode 4 Transponder \\
\hline Cost & $\$ 7995.00$ & $\$ 2700$ & -- \\
\hline Frequency & $978 \mathrm{MHz}$ & $1090 \pm 1 \mathrm{MHz}$ & $118-137 \mathrm{MHz}$ \\
\hline Weight & -- & $0.75 \mathrm{~kg}$ & $2.8 \mathrm{~kg}$ \\
\hline Size $(\mathrm{cm})$ & $8.9 \times 18.8 \times 32$ & $3.9 \times 12.4 \times 17.4$ & $13 \times 22 \times 80$ \\
\hline GNSS & WAAS enabled GPS & Internal GNSS receiver & Internal GNSS receiver \\
\hline Data Rate & Up to 1 Mbps & Up to 1 Mbps & Up to 19.2 Kbps \\
\hline Transmit Power & 40 Watts & 20 Watts & -- \\
\hline Limitations & US market only & Only ADS-B out (receiver also needed) & -- \\
\hline
\end{tabular}

The systems listed in Table 5 are designed for general aviation aircraft making them affordable and relatively simple to install and operate. Each of the systems has advantages and disadvantages that are inherent to the underlying technology (i.e. UAT vs. Mode S ES vs. VDL Mode 4). The $1090 \mathrm{MHz}$ Mode S ES solution will probably be the most widely used solution even though there is criticism that the frequency spectrum is already overused. Regardless, with the availability of low-cost general aviation equipment, ADS-B is a promising communication solution for an OCT network. All flying objects in the atmosphere (aircraft or HAP) are required to have on-board equipment to interface with air traffic control. Additionally, ADS-B equipment can be mounted on ground vehicles to facilitate ground safety. Adding ADS-B capability to this equipment is relatively easy and inexpensive. In addition, using the same equipment for ATC surveillance and OCT terminal-to-terminal communication may reduce overall terminal weight and cost. For example, the DLR Capanina Trial 2 weather balloon used a Microair T2000 transponder. This transponder cost $\$ 2,300$ and weighed $0.6 \mathrm{~kg}$ [1]. For a slightly higher price and weight, the Filser TRT800 could have been used that had the capability to transmit ADS-B information. 


\section{TEST SCENARIO AND INITIAL TEST RESULTS}

\subsection{Test overview and objectives}

Based on the systems and concepts discussed in Section 3 and Section 4, one position measurement system and one communication system were chosen for testing within the framework of several ongoing projects. The Crossbow NAV420 was selected due to its cost, proven performance, and flexibility (see Fig. 4, left). For the current projects, it was important to pick a position measurement system that was not susceptible to intermittent signal losses and the resulting position data (attitude and location) losses. This precluded the use of a GNSS-only solution due to the possible signal losses when the antennas are blocked. An ADS-B communication system was chosen based on the low cost, and the concept originality, and the potential for leveraging a current aircraft system for the OCT communication task. A Filser RTH60 1090MHz ADS-B receiver was chosen for the test (see Fig. 4, right).
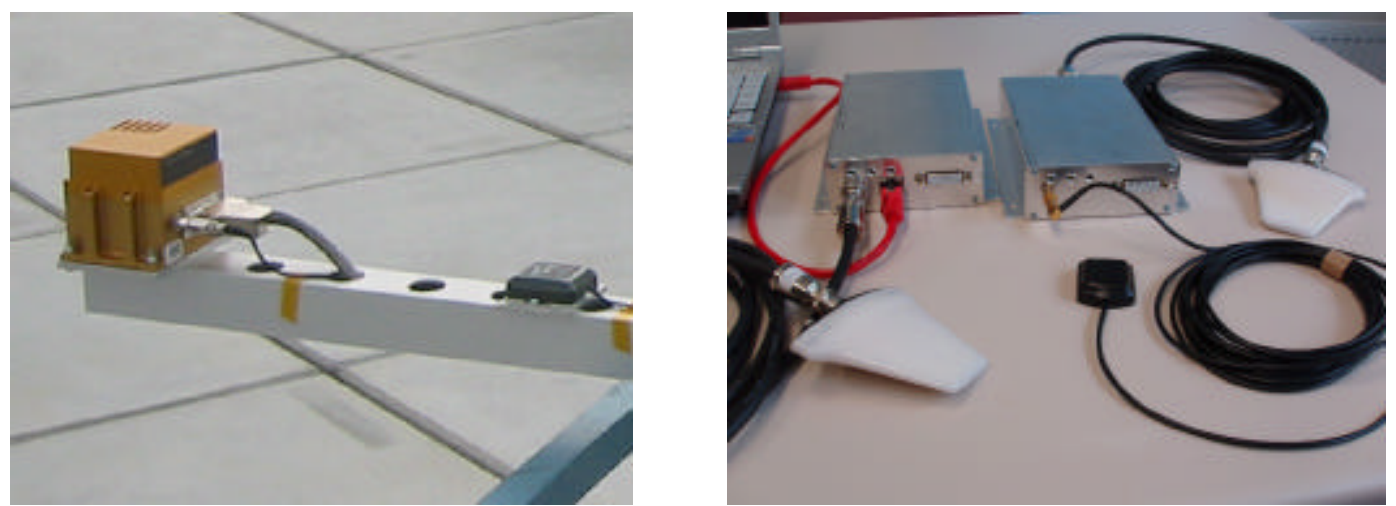

Fig. 4 Left: Crossbow NAV420 GPS-aided inertial system integrated into optical terminal. Right: Filser RTH60 ADS-B receiver, TRG80 ADS-B transmitter, and supporting equipment.

The overall objective of the test was to demonstrate promising technologies and concepts (i.e. the NAV420 and an ADSB communication system) that could be applied to future OCT networks or OCT test programs. This was done by integrating these two components into a typical optical communication scenario. The test scenario is similar to that shown in Fig. 3. At the ground station, the NAV420 and the RTH60 were integrated into an optical terminal. For mobile terminals, the test took advantage of local aircraft traffic. If an aircraft was equipped with a GNSS receiver and an ADSB capable transponder, it was a "target of opportunity" and could be tracked by the ground station. Although the actual aircraft position data were not available for analysis, it was possible to empirically evaluate the tracking system

\subsection{Test procedure}

The ground station was set up on top of a building located $0.6 \mathrm{~km}$ away from the DLR Oberpfaffenhofen airfield. ADS-B capable aircraft flying near the airfield transmitted ADS-B position messages which were received and processed by the RTH60 receiver. The RTH60 decoded the messages and sent the data to a laptop over an Ethernet connection via User Datagram Protocol (UDP) packets. The laptop ran the user interface and analyzed/displayed the data in real time. The data from a selected aircraft were reformatted and sent to the tracking computer via COM port.

The Crossbow NAV420 GPS-aided inertial system provided location and attitude data to the tracking computer. Using the ADS-B target information and the NAV 420 position information, the tracking computer calculated the correct pointing angles and drove the coarse pointing assembly motors, thereby pointing the ground station tracking camera toward the aircraft.

All of the software was written using LabVIEW from National Instruments. LabVIEW was chosen in order to be compatible with other real time software developed within the Optical Communication Group at DLR.

\subsection{Results}

Initial tests were done using only the RTH60 receiver to ensure that the ADS-B message decoding was being done properly. Fig. 5 shows a screen shot from the laptop running the analysis/display software. The display shows the track of an aircraft flying in the pattern at the DLR Oberpfaffenhofen airfield. 


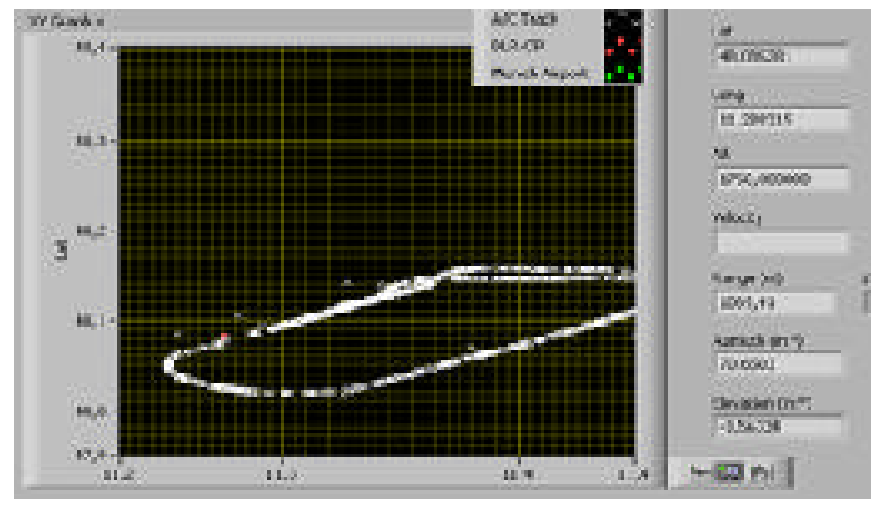

Fig. 5 Screen shot of the ADS-B LabVIEW display showing an aircraft in the pattern at the DLR Oberpfaffenhofen airfield

After the RTH60 receiver performance was verified, tracking tests with the full system were accomplished. Fig. 6 shows pictures from the optical terminal tracking camera showing an ADS-B enabled aircraft being tracked using the ADS-B receiver data.
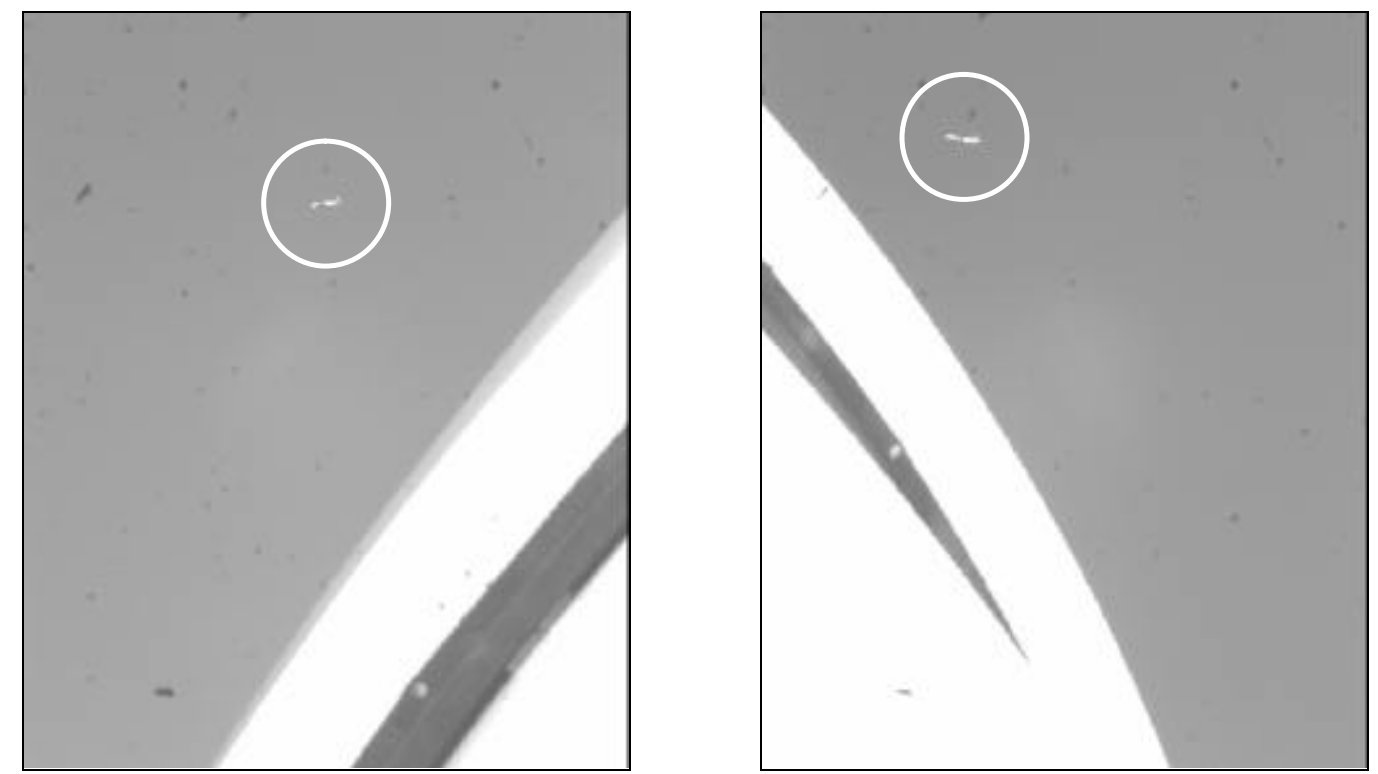

Fig. 6 Pictures of an aircraft being tracked by the optical terminal as the aircraft passes by the dome of the DLR Optical Ground Station. The aircraft is located within the white circle. The narrow dimension represents a $17^{\circ}$ field of view

Qualitative observations indicated that the aircraft could normally be held within the $17^{\circ}$ field of view of the tracking camera. Due to the large tracking camera FOV, the low heading accuracy $\left(3^{\circ}\right)$ of the NAV 420 did not impact this test. However, there are scenarios that would require better pointing accuracy, or, in which better heading accuracy would significantly increase PAT system performance. Two communication system deficiencies were observed during the tracking tests and data analysis: 1) the ADS-B position update rate was not frequent enough for low altitude aircraft; 2) the system occasionally received an incorrect/corrupted position message, which was passed on to the tracking system.

The maximum position transmission rate is $2 \mathrm{~Hz}$ assuming the receiver correctly receives and decodes every message. The effective transmission rate is lower when corrupted messages are discarded or a message is not received. This measurement delay was evident while tracking the aircraft and was observed as a lag in the tracking system. When the tracking system received an updated position, it would move to put the aircraft in the center of the tracking camera picture. The aircraft then moved from the center of the picture until another position update was received. The receiver also occasionally received incorrect ADS-B messages. No error detection algorithms were implemented in the decoding software, so these errors were sent to the tracking system. When this happened, the terminal would attempt to point to the incorrect position location, losing sight of the aircraft. 


\subsection{Tracking and prediction filter simulation}

In order to improve the tracking system deficiency caused by the low ADS-B transmission rate, a tracking and prediction filter was developed, namely, a g-h (or $\alpha-\beta)$ filter. The g-h filter can estimate the future position and velocity of a vehicle based on current and past measurement data. Equation 2 and 3 show the gh tracking-filter equations that are used extensively in radar tracking systems [29].

$$
\begin{aligned}
& {\underset{x}{n+1, n}}_{\mathbb{*}}^{*}=x_{n, n-1}^{*}+\frac{h_{n}}{T}\left(y_{n}-x_{n, n-1}^{*}\right) \\
& x_{n+1, n}^{*}=x_{n, n-1}^{*}+T x_{n+1, n}^{*}+g_{n}\left(y_{n}-x_{n, n-1}^{*}\right)
\end{aligned}
$$

where, $\mathcal{X}_{n+1, n}^{*}$ and $x_{n+1, n}^{*}$ are the estimated velocity and position of the target at future time $n+1$

$\mathbb{R}_{n, n-1}^{*}$ and $x_{n, n-1}^{*}$ are the estimated velocity and position at the current time, $n$, based on past data (it is assumed that the present target velocity and position can not be exactly measured, so a filtered estimate is calculated based on previous measurement data and previous estimates).

$T$ is the time interval at which the position and velocity predictions are made.

$y_{n}$ is the received (or measured) target position at the current time (i.e. at time, $n$ ).

$h_{n}$ and $g_{n}$ are parameters used to weight the filtered estimates based on the accuracy of the measurements and past filtered results

These equations are called prediction equations because they predict the position and velocity of the aircraft at some time in the future. For these equations a constant-velocity assumption is made which is a reasonable one as long as the time between observations $\mathrm{T}$ is small or the target acceleration is small. An in depth derivation of these equations can be found in [29].
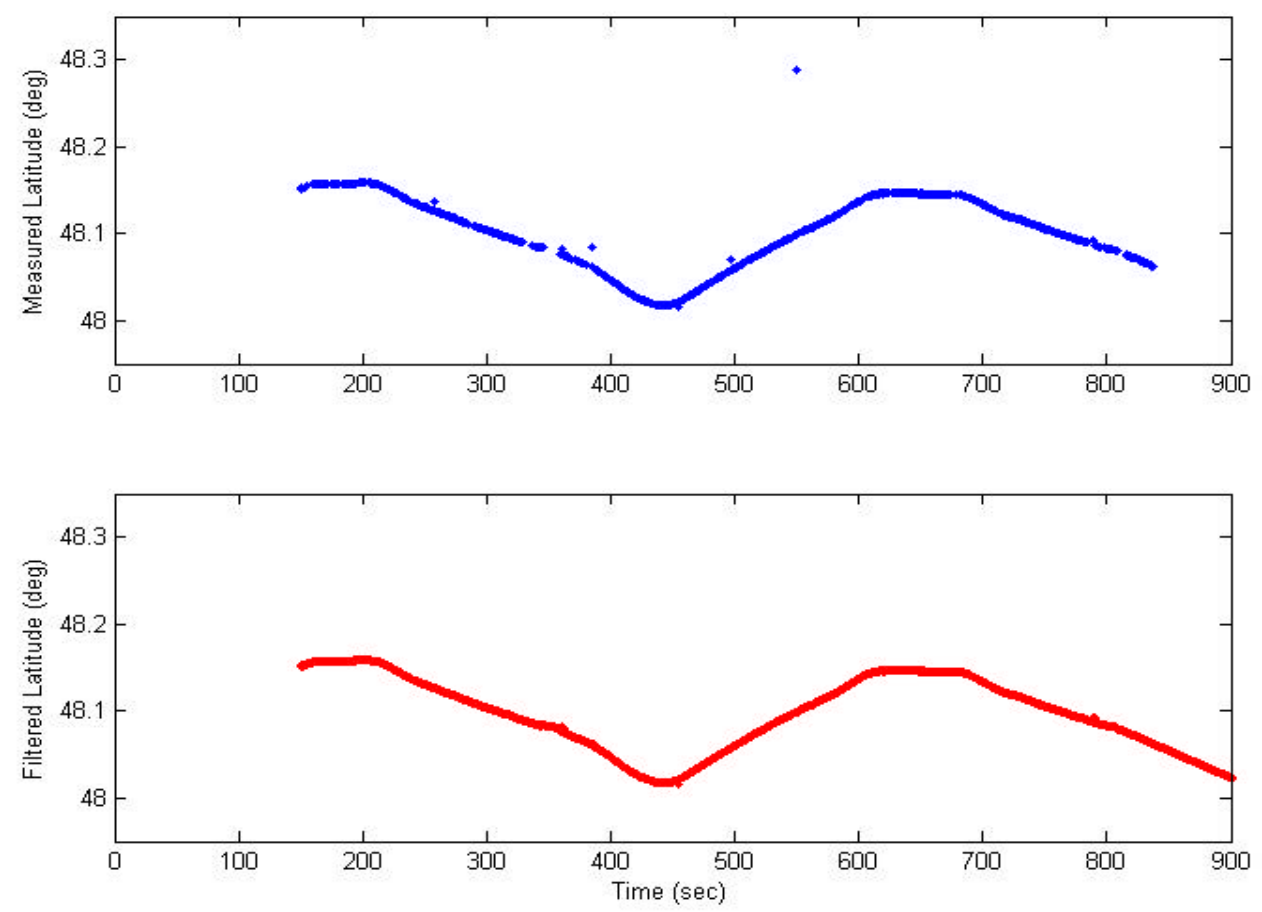

Fig. 7 Top: Raw latitude data received by RTH60 and processed using LabVIEW message decoding software; Bottom: g-h filtered latitude data $(\mathrm{g}=0.9 ; \mathrm{h}=0.01, \mathrm{~T}=100 \mathrm{~ms})$ 
In conjunction with the filter, an error checking algorithm was created to remove invalid ADS-B messages by comparing the new position report to the predicted position and applying an acceptance tolerance of approximately $1 \mathrm{~km}$ (i.e. if the measured position was greater than $1 \mathrm{~km}$ away from the predicted position, the measurement was not used). The filter and error checking algorithm were applied to actual tracking data received by the RTH60 receiver. Fig. 7 and Fig. 8 show results from this simulation.
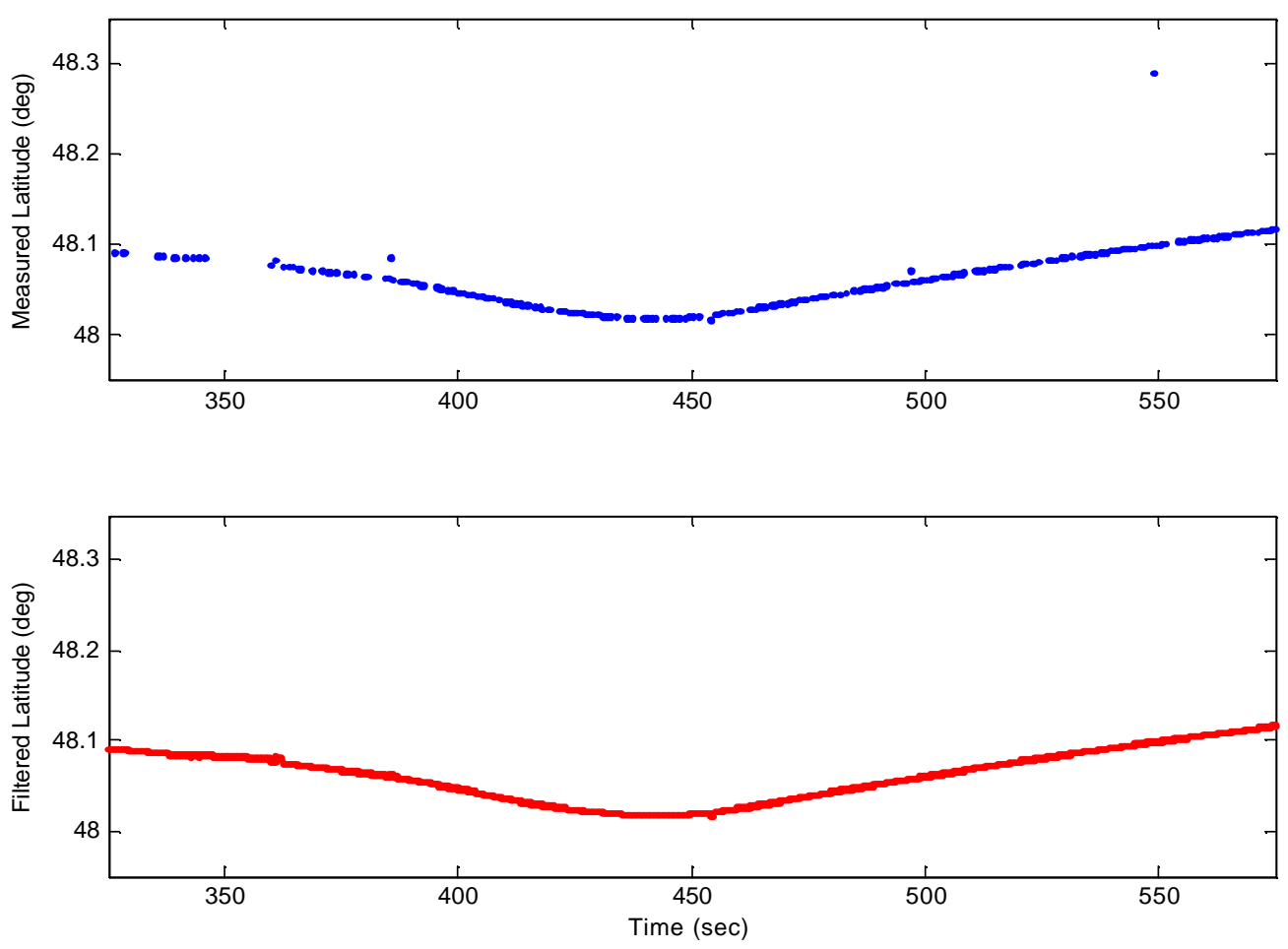

Fig. 8 Top: Detailed view of raw latitude data received by RTH60 and processed using LabVIEW message decoding software; Bottom: Detailed view of g-h filtered latitude data $(\mathrm{g}=0.9 ; \mathrm{h}=0.01, \mathrm{~T}=100 \mathrm{~ms})$

The simulation results show that it is possible to reduce the effects of the relatively large period between measurements in the ADS-B communication system. Using the predictive filter, the gaps in the measurements caused by corrupted or missing position data could be filled in (e.g. Fig. 8 at approximately $\mathrm{t}=350$ seconds. Additionally, error detection algorithms made it possible to eliminate erroneous position measurements (e.g. Fig. 8 at approximately $\mathrm{t}=380,500$ and 550 seconds).

\section{CONCLUSION AND RECOMMENDATIONS}

This paper discussed promising technologies and techniques that will aid the pointing, acquisition, and tracking (PAT) tasks in optical communication terminals (OCT). Based on a discussion of factors impacting PAT accuracy, position measurement and inter-terminal communications were identified as important systems. The highlights of a feasibility study focusing on potential hardware and techniques for these two systems was presented. Based on the results of the study, a position system and a communication system were integrated into an OCT and tested.

Test results showed that both devices worked well and have the potential to be used in OCTs. However, the performance of both systems could be improved. To reduce tracking errors arising from the communication system, further research should be done on prediction/tracking filters and error checking algorithms. Tests should be carried out to validate these improvements in OCT scenarios. To decrease tracking errors based on position measurement errors from the GNSSaided inertial system, a 2-antenna system should be researched and tested. 


\section{REFERENCES}

1. J. Horwath, M. Knapek, B. Epple, M. Brechtelsbauer, B. Wilkerson. "Broadband Backhaul Communication for Stratospheric Platforms: Results of the Stratospheric Optical Payload Experiment," Proceedings of SPIE Optics and Photonics 2006, San Diego.

2. M. Knapek, J. Horwath, N. Perlot, K. Zettl, D. Giggenbach, B. Wilkerson. "Transportable Optical Ground Station for Free-Space Laser Communications," Proceedings of SPIE Optics and Photonics 2006, San Diego.

3. B. Epple. "Using a GPS-Aided Inertial System for Coarse-Pointing of Free-Space Optical Communication Terminals," Proceedings of SPIE Optics and Photonics 2006, San Diego.

4. Galileo press release: "GALILEO and GPS will navigate side by side: EU and US sign final agreement" http://europa.eu.int/comm/dgs/energy_transport/galileo/documents/press_en.htm

5. The Galilei Project; GALILEO Design Consolidation brochure

(http://europa.eu.int/comm/dgs/energy_transport/galileo)

6. Garmin WAAS webpage (http://www.garmin.com/aboutGPS/waas.html)

7. Bradford W. Parkinson (Ed), James J., Jr. Spilker (Ed). Global Positioning System: Theory \& Applications (Volume Two). 1996

8. Daniel J. Biezad. Integrated Navigation and Guidance Systems. QED Educational Services. 2000.

9. Christopher Hide, Terry Moore, Martin Smith. GPS and low cost INS integration using commercial off-the-shelf components. Institute of Engineering, Surveying and Space Geodesy, University of Nottingham

10. E-mail from Robert Pinto, Enpoint President and company website, http://www.enpoint.com/Products/products.html

11. Thales ADU5 Data sheet (http://products.thalesnavigation.com/en/products/product.asp?PRODID=899)

12. Setentrio PolaRx2@ Data Sheet (http://www.septentrio.com/products_polarx2at.htm)

13. Crossbow NAV420 Data sheet (http://www.xbow.com/Products/productsdetails.aspx?sid=104)

14. Javad Navigation Systems webpage (http://www.javadgps.com/)

15. E. Lutz, M. Werner, A. Jahn. Satellite Systems for Personal and Broadband Communications. Springer, 2000.

16. GMPCS Personal Communications Website (http://www.gmpcs-us.com/)

17. W. V. Jones, et.al. "Ultra Long Duration Ballooning Technology Development" 29th International Cosmic Ray Conference, Pune, 2005.

18. NAL Research Company Webpage (http://www.nalresearch.com/ApplicationDescriptions.html)

19. Andy Dornan "Mobile Satellite Services: From Third World to Third Generation"

20. W. David Gardner (TechWeb.com) "In Wake Of Hurricanes, Inmarsat Pitches New Satellite Service” Sept. 28, 2005 http://informationweek.com/story/showArticle.jhtml?articleID=171201324

21. Andy Giegerich, "Anatomy of a deal" The Business Journal of Portland, Oct. 2, 2005

22. E-plus Online Shop (www.eplus.de)

23. ADS-B webpage (http://www.ads-b.com/home.htm)

24. ICAO Eleventh Air Navigation Conference, Montreal, 22 September to 3 October 2003 AN-Conf/11-IP/12 COMPARATIVE ANALYSIS OF ADS-B LINKS

25. ICAO Eleventh Air Navigation Conference, Montreal, 22 September to 3 October 2003 AN-Conf/11-WP/6 AUTOMATIC DEPENDENT SURVEILLANCE — BROADCAST CONCEPT OF USE

26. Garmin Webpage (http://www.garmin.com/products/gd190/)

27. Filser GmbH webpage (http://www.filser.de/)

28. C.N.S Systems webpage (http://www.cns.se/vdl4000ga.php)

29. Eli Brookner. Tracking and Kalman Filtering Made Easy. John Wiley \& Sons, Inc. 1998.

30. European Community $6^{\text {th }}$ Framework Program: ATENAA, Advanced Technologies for Networking in Avionic Applications. CEC contract number AST3-CT-2004-502843, www.atenaa.org. 